\title{
Anomalous Bumpy Structures in the Capture Cross Sections of Antiprotons by Helium
}

\author{
X. M. Tong, ${ }^{1,2, *}$ K. Hino, ${ }^{1,3,2}$ and N. Toshima ${ }^{1}$ \\ ${ }^{1}$ Doctoral Program in Materials Science, Graduate School of Pure and Applied Sciences, University of Tsukuba, \\ 1-1-1 Tennodai, Tsukuba, Ibaraki 305-8573, Japan \\ ${ }^{2}$ Center for Computational Sciences, University of Tsukuba, 1-1-1 Tennodai, Tsukuba, Ibaraki 305-8577, Japan \\ ${ }^{3}$ Doctoral Program in Frontier Science, Graduate School of Pure and Applied Sciences, University of Tsukuba, \\ 1-1-1 Tennodai, Tsukuba, Ibaraki 305-8573, Japan
}

(Received 15 February 2008; published 13 October 2008)

\begin{abstract}
We investigate the state-specified capture process of antiprotons by helium. Freezing one of the two electrons, we reduce this four-body rearrangement problem into a three-body problem. The capture cross sections are calculated by solving the Chew-Goldberger-type integral equation. Differing from the capture of antiprotons by hydrogen atoms, the bumpy structures are revealed in the total angular momentum dependent capture cross sections. Further analysis shows that the bumps arise from the partial channel closing due to the removal of the energy degeneracy in the antiprotonic helium.
\end{abstract}

DOI: 10.1103/PhysRevLett.101.163201

PACS numbers: $34.70 .+\mathrm{e}, 25.43 .+\mathrm{t}, 36.10 .-\mathrm{k}$

The interaction of antiprotons $(\bar{p})$ with matter is one of the hot topics in recent atomic physics since the discovery of long lifetime antiprotonic helium [1,2] and the production of cold antihydrogen atoms [3,4]. The capture of antiprotons by helium is a typical Coulomb four-body rearrangement problem. The full quantal and nonperturbative solution of this problem is still out of the reach of the current high-power supercomputers. Thus, several groups have studied the capture of antiprotons by hydrogen atoms, which is a Coulomb three-body rearrangement problem, by various approaches such as the classical trajectory Monte Carlo (CTMC) method [5], the time-dependent wave packet (TDWP) method [6], and other quantum methods $[7,8]$. The state-specified capture cross sections of antiprotons by hydrogen atoms have been obtained recently by a time-dependent method $[9,10]$. The advantage of this method is that by rewriting the timeindependent scattering equation into a time-dependent one, the complicated boundary condition is converted into an initial condition which can be easily imposed. Although such theoretical studies provide an understanding of the capture processes, it is very difficult to compare the calculations with the helium experiments quantitatively.

Recently, the capture cross sections of antiprotons by helium ions were calculated by Cohen [11] using the CTMC method and by Sakimoto [12] using the TDWP. However, the results differ from each other significantly, especially in the low collision energy region. Thus, the applicability of the classical mechanics at such low energies seems doubtful, especially for the motion of the electron. Furthermore, while the total capture cross sections of antiprotons by many-electron atoms have been obtained by the CTMC method $[5,13]$, the state-specified capture cross sections have not been provided yet. Meanwhile, the low energy antiproton beam (around $30 \mathrm{eV}$ ) will be available for the experiment to study the capture of antiprotons by helium soon [14]. The statespecified capture cross sections are very important to analyze the decay processes of antiprotonic helium. Unfortunately, such information is not available yet. In this Letter, we will report our theoretical studies on the state-specified capture of antiprotons by helium.

Because of the numerical difficulty, we have to adopt various approximations to make the problem solvable. The primary approximation here is to assume that one of the two electrons in the helium is considered passive and frozen into the ground state of the complex of the helium nucleus and antiproton. Within this approximation, the problem is reduced to the tractable Coulomb three-body problem, which can be solved by the method recently developed by us for the capture of antiprotons by hydrogen atoms $[9,10]$. To our surprise, unlike the smooth total angular momentum $(L)$ dependent capture cross sections by hydrogen atoms, the $L$-dependent capture cross sections by helium show anomalous bumpy structures. Further studies show that the bumps arise from the partial channel closing due to the removal of the energy degeneracy in the antiprotonic helium. For the convenience of later discussion, we define a channel as a combination $(n, \ell)$, where $n$ and $\ell$ are the principle and angular momentum quantum numbers of the antiproton bound to the helium ion, respectively.

The Hamiltonian of the collision system with a passive electron frozen into the ground state of the complex of the helium nucleus and antiproton can be written as (atomic units, $m=e=\hbar=1$, are used hereafter unless otherwise stated) 


$$
\begin{aligned}
H \approx & \left(-\frac{1}{2 \mu_{p}} \nabla_{\mathbf{R}}^{2}+U(R)\right)+\frac{1}{|\mathbf{R}-\mathbf{r}|} \\
& +\left(-\frac{1}{2 m_{e}} \nabla_{\mathbf{r}}^{2}+V_{\mathrm{eff}}(r)\right),
\end{aligned}
$$

where $\mu_{p}$ is the reduced mass between the helium nucleus and antiproton, $m_{e}$ the mass of electron, and $\mathbf{R}$ and $\mathbf{r}$ the vectors from the helium nucleus to the antiproton and the active electron, respectively. We choose the helium nucleus as the center of the coordinates. The mass polarization effect is not important in the low energy region in such coordinates. $U(R)$ is the effective potential of the antiproton moving in the combined field of the helium nucleus and the passive electron in the ground state and it can be calculated from the following equation adiabatically,

$$
H_{a} \Psi\left(\mathbf{r}_{b} ; R\right)=U(R) \Psi\left(\mathbf{r}_{b} ; R\right),
$$

with $\Psi\left(\mathbf{r}_{b} ; R\right)$ the associated adiabatic wave function, $\mathbf{r}_{b}$ the coordinates of the passive electron and

$$
H_{a}=\left(-\frac{1}{2 m_{e}} \nabla_{\mathbf{r}_{b}}^{2}-\frac{2}{r_{b}}+\frac{1}{\left|\mathbf{R}-\mathbf{r}_{\mathbf{b}}\right|}-\frac{2}{R}\right) .
$$

$V_{\text {eff }}(r)$ is the effective potential to describe the active electron interacting with the $\mathrm{He}^{+}$ion. We choose $V_{\text {eff }}(r)$ as a six-parameter model potential [15], which is calculated from the density functional theory with the optimized effective potential [16]. Such an approximated Hamiltonian $H$ ensures the correct forms for the initial channel (a helium atom and a free antiproton) as well as for the final channel (an antiprotonic helium and a free electron). Within the above approximations, the state-specified capture processes of antiprotons by helium are analyzed in the similar way to the case of the capture processes of antiprotons by hydrogen atoms just by replacing the antiproton-proton interaction and the electron-proton interaction in the latter by $U(R)$ and $V_{\text {eff }}(r)$, respectively. Note that the validity of using an adiabatic potential $U(R)$ has been discussed in Ref. [17]. Here, we define the $\mathrm{He}^{+}$ ground state energy as zero for $U(R)$ when $R \rightarrow \infty$. The Hamiltonian of the incident channel and the corresponding interaction are now expressed as

$$
\begin{gathered}
H_{i}=-\frac{1}{2 m_{e}} \nabla_{\mathbf{r}}^{2}+V_{\mathrm{eff}}(r)-\frac{1}{2 \mu_{p}} \nabla_{\mathbf{R}}^{2}, \\
V_{i}=\frac{1}{|\mathbf{R}-\mathbf{r}|}+U(R),
\end{gathered}
$$

and the initial wave function is

$$
\Psi_{i}(\mathbf{r}, \mathbf{R})=\psi_{1 s}(\mathbf{r}) e^{i \mathbf{k}_{0} \cdot \mathbf{R}} .
$$

Here, $\psi_{1 s}$ is the helium $1 s$ wave function with the eigen energy $\epsilon_{1 s}$ and $\mathbf{k}_{0}$ is the initial momentum of the antiproton in the center-of-mass frame. The incident energy is given by $E_{c}=\mathbf{k}_{0}^{2} / 2$. The scattering wave function is of the form of the time integral equation as

$$
\Psi^{+}(0)=\Psi_{i}-i \int_{-\infty}^{0} e^{i(H-E) t} e^{\eta t} V_{i} \Psi_{i} d t
$$

with $\eta$ a positive infinitesimal number to represent the adiabatical switch on of the interaction $V_{i}$ and $E=E_{c}+$ $\epsilon_{1 s}$, the total energy of the system. The time integral of Eq. (6) is performed using the partial wave expansion and the details can be found in our previous works $[9,10]$. With the scattering wave function $\Psi^{+}(0)$ in hand, we can obtain all the detailed information of the collision system, including the state-specified capture cross sections.

Figure 1 shows the total angular momentum $L$-dependent capture cross sections (the solid curve). As $L$ increases, the capture cross sections first increase in an irregular manner, reach the maximum at $L=38$, and then, drop sharply. This global feature is similar to the capture of antiprotons by hydrogen atoms. Unlike the hydrogen case, however, the present cross sections show the anomalous bumpy structures. In Fig. 1, we also plotted the partial contributions when the ejected electron is in different angular momentum states, namely, $\ell_{e}=0$ or 1 . Because the contribution from the $\ell_{e}=0$ channel dominates the overall cross sections, the anomalies appearing in the cross sections are closely related to the dips appearing in the partial cross sections for $\ell_{e}=0$ (depicted by the dashed curve). In other words, the antiproton is mainly captured into states with the angular momentum $\ell$ equals the total angular momentum $L$. By the way, it is notable that the present calculations seem more difficult than those of the hydrogen case due to the larger reduced mass $\mu_{p}$ and the larger effective nuclear charge of helium. To check the numerical accuracy, the simulation conditions such as the number of grids, the size of the space, and so on, have been varied. As a result, it is convinced that the anomalies manifested in Fig. 1 are insensitive to the changes of these parameters. Thus, the anomalies are ascribable to a certain physics, not due to the numerical inaccuracy.

Although the capture process of the antiprotons by hydrogen atoms and helium atoms are similar to each other within the present approximation, the energy structures of

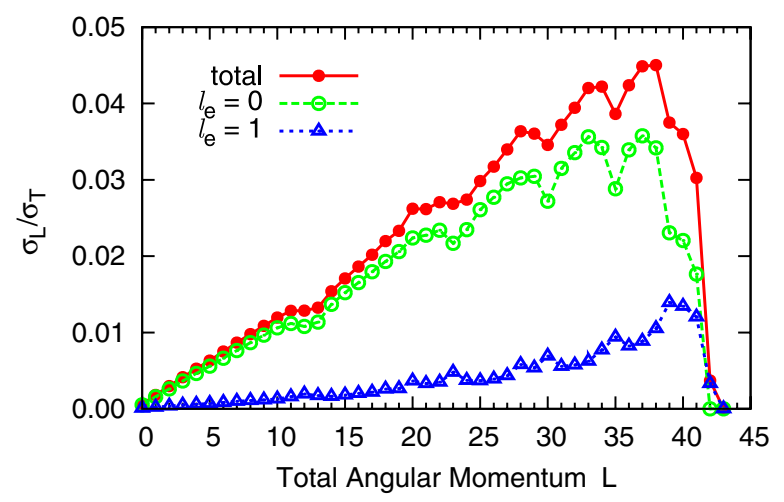

FIG. 1 (color online). The $L$-dependent capture cross sections of antiprotons by helium at $10 \mathrm{eV}$ incident energy. 
protonium $(\bar{p} p)$ and antiprotonic helium $\left(\bar{p} \mathrm{He}^{+}\right)$are different. Replacing the Coulomb potential in the former by the effective potential $U(R)$ in the latter, the energy degeneracy assigned by $n$ and $\ell$ is removed. Figure 2 shows the energy structures of antiprotonic helium. The bound states in Fig. 2 are embedded in the continuum states with the antiproton in a lower excited state and the electron in a continuum state. So the bound states are actually autoionized and the formed highly excited antiprotonic helium can decay into a H-like protonium due to the Auger process. Since we focus on the first step, the formation of the antiprotonic helium, we will ignore the decay processes. Apparently, for a given $n$, the energy increases as the $\ell$ increases. Lower $\ell$ states can penetrate into the deeper inner region than higher $\ell$ states due to its lower centrifugal potential barrier, resulting in lower binding energies for the lower $\ell$ states with the same $n$.

As shown in Fig. 2, the energy curve for a given $n$ and different $\ell$ crosses over with the total energy (dashed straight line in Fig. 2). The crossover positions are in good agreement with the dip positions in the total $L$-dependent capture cross sections in Fig. 1 . Let us focus on $n=47$ as an example to show the origin of the dips. With the $10 \mathrm{eV}$ incident energy, the antiprotons can be captured to $n=47$ and $\ell \leq 11$ due to the energy conservation. This means that this channel is open for the capture to $\ell \leq 11$ states and is closed for the capture to $\ell \geq 12$ states. This fact would allow us to speculate that the manifestation of the anomaly around $L \sim l=12$ is attributed to the channel closing of the states with $l \geq 12$. Here, the channel closing means that for a given $n$ the capture process is forbidden when the $\ell$ is larger than a critical value. This speculation is more clarified by comparing the situations between the cases of the hydrogen and helium targets. In the former, all channels with $n=47$ and different $\ell$ 's contribute to the capture cross sections because of the energy degeneracy, and the capture cross sections as a function of $L$ show no anomaly. In contrast to this, in the latter, the states with $n=47$ and $l>11$ do not contribute

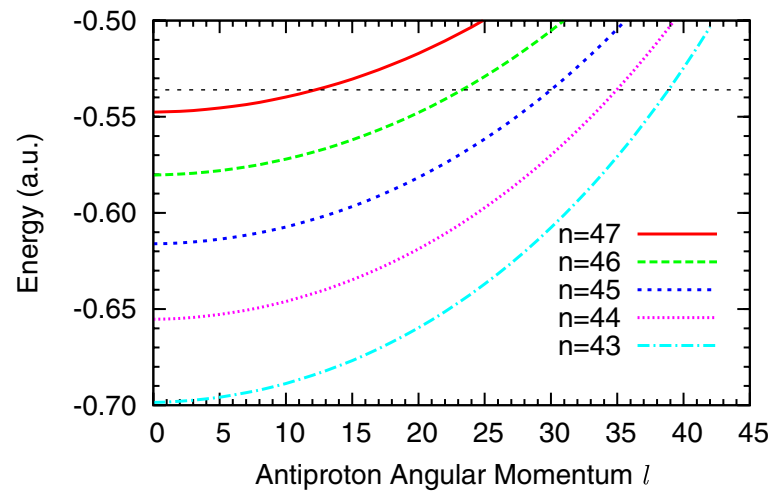

FIG. 2 (color online). The energy levels of antiprotonic helium. The horizontal dashed line is the total energy for $10 \mathrm{eV}$ incident case. to the capture cross sections, leading to the dip. In a similar way, it is shown that the next dip corresponds to the closing of channel 46 at $L=23$, and so on. In general, the partial inelastic scattering cross sections exhibit cusps when the incident energies cross a new threshold since the cross sections differ significantly for the energies below and above the threshold. Here we see the cusps from a different aspect, i.e., the cross sections as a function of the $\ell$ for a given incident energy and $n$.

Figure 3 shows the state-specified capture cross sections, where the gradated trace is depicted in the $(n, \ell)$ plane. The antiprotons are mostly captured to the region around $n \sim 42$ and $\ell \sim 38$ states, which is much higher than that of the hydrogen case.

From our present calculation, we obtain all the detailed capture cross sections $\sigma_{n \ell}^{L}$. In Fig. 4 we show the ejected electron energy distribution, given by,

$$
P(\epsilon)=\frac{d \sigma}{d \epsilon}=\sum_{L, n, \ell} \delta\left(\epsilon-\epsilon_{L n \ell}\right) \sigma_{n \ell}^{L} .
$$

Here $\epsilon_{L n \ell}$ is the ejected electron energy associated with the antiproton captured to the $(n, \ell)$ states with the total angular momentum $L$. The $\delta$-function is replaced by $\delta(x)=\exp \left(-x^{2} / \tau^{2}\right) /(\tau \sqrt{\pi})$ with $\tau$ the energy resolution. The details of the convoluted spectra depend on the energy resolution. As an example, we choose $\tau=0.5 \mathrm{eV}$. In Fig. 4, we see that the ejected electrons mainly distribute in the low energy region $(<10 \mathrm{eV})$ and peak around $1 \mathrm{eV}$. For lower incident energy $\left(E_{c}=2.0 \mathrm{eV}\right)$, plateau structures appear around 3, 5, and $8 \mathrm{eV}$. Further analysis shows that such structures are associated with the anomalies discussed above regarding Figs. 1 and 2 in the capture cross sections. For the higher incident energy $(20 \mathrm{eV})$, the ejected electron energy distribution changes smoothly, implying that the anomaly is less significant for the high incident energy. This observation is due to the fact that the antiproton can be captured into higher $(n, \ell)$ states with denser energy levels, where the channel closing contribu-

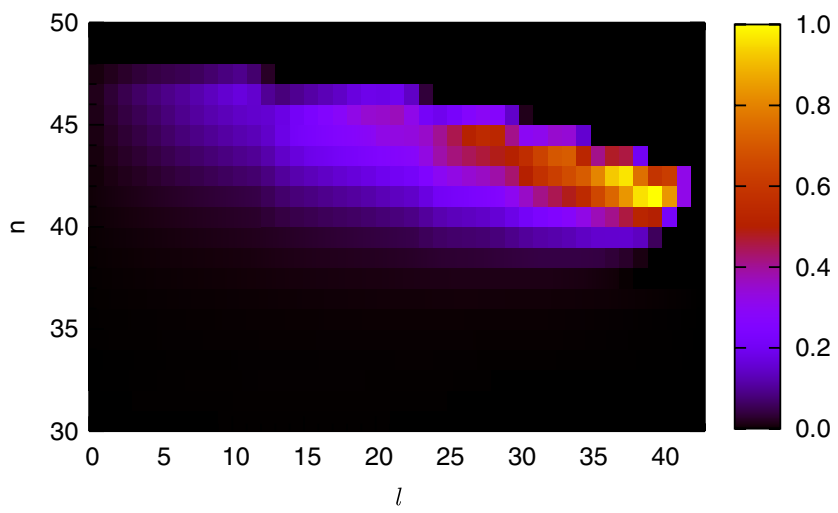

FIG. 3 (color online). The state-specified capture cross sections normalized to the total capture cross section for $10 \mathrm{eV}$ incident energy. 


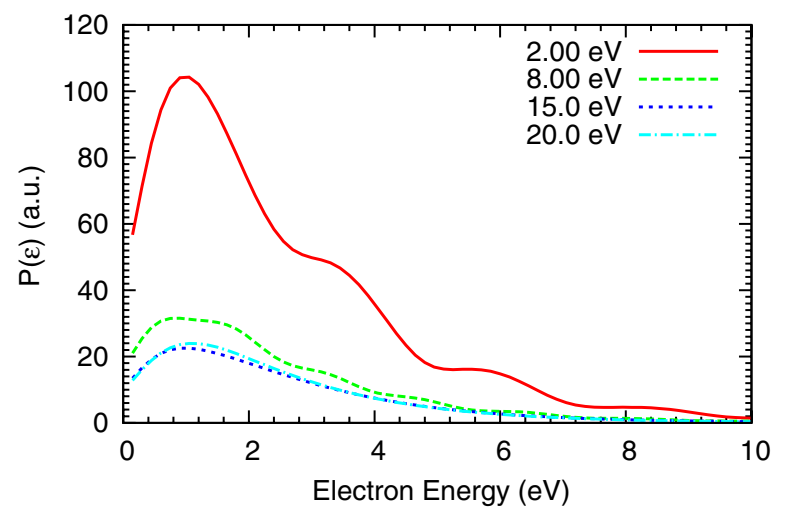

FIG. 4 (color online). The ejected electron energy distributions due to the capture of antiprotons by helium at different incident energies.

tion is less important. The energy distribution $P(\epsilon)$ is expected to be measured in the future experiment [14]. The measured electron spectra could also possibly arise from the Auger decay of the antiprotonic helium which is ignored here.

Finally, we compare our total capture cross sections with the existing results obtained by the CTMC method. Figure 5 shows the cross sections as a function of the incident energy. Our cross sections are between those by helium atoms and $\mathrm{He}^{+}$ions calculated by CTMC method $[11,13]$. Our results are in agreement with the CTMC results in the relatively higher energy region $\left(E_{c} \sim\right.$ $20 \mathrm{eV}$ ), whereas in the lower energy, both of the CTMC results are larger than ours. It is inferred that this discrepancy is due to either (a) the failure of the CTMC method in such a low incident energy region or (b) the appreciable contribution of the capture-induced excitation of the bound electron. The second issue suggests the possibility of breakdown of the passive electron approximation assumed throughout this Letter. It will be necessary to implement the calculations incorporating the capture-induced excitation process in the future. The anomalies observed in the present calculations are associated with the removal of the energy degeneracy and this phenomenon should exist even in the full quantal nonperturbative calculation without any approximations.

To summarize, we have studied the state-specified capture processes of antiprotons by helium in the low collision energy region by approximating the Coulomb four-body rearrangement process to the associated three-body rearrangement process. The total angular momentum dependent capture cross sections by helium show anomalous behavior due to the nondegenerate energy levels of the

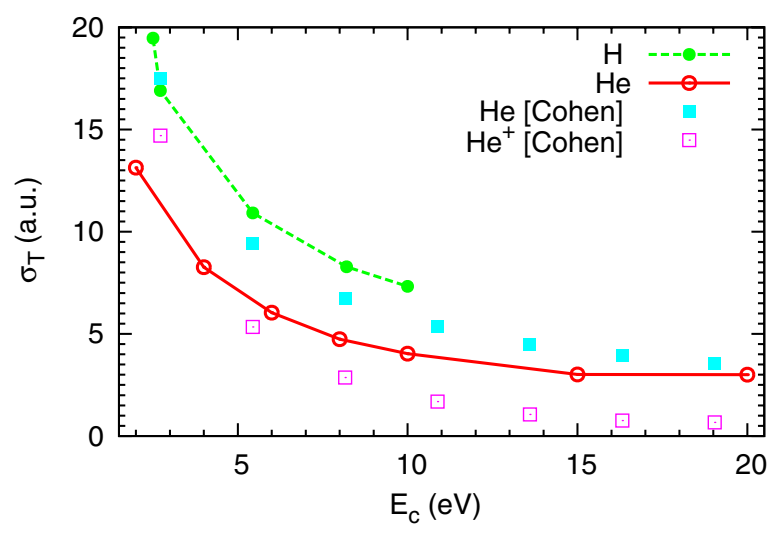

FIG. 5 (color online). The total capture cross sections of antiprotons by helium atoms (solid curve) and hydrogen atoms (dashed curve). The capture cross sections by helium atoms (filled squares) and helium ions (open squares) calculated by the CTMC method [11,13] are also plotted.

antiprotonic helium for the given principle quantum number $n$ and different angular momentum $l$. More specifically, the anomalies originate from the channel closing due to the energy conservation.

This work was supported by Grant-in-Aids for Scientific Research (B) and (C) from the Japan Society for the Promotion of Science.

*tong@ims.tsukuba.ac.jp

[1] M. Iwasaki, et al., Phys. Rev. Lett. 67, 1246 (1991).

[2] T. Yamazaki, N. Morita, R. S. Hayano, E. Widmann, and J. Eade, Phys. Rep. 366, 183 (2002).

[3] M. Amoretti et al., Nature (London) 419, 456 (2002).

[4] G. Gabrielse et al., Phys. Rev. Lett. 89, 213401 (2002).

[5] J.S. Cohen, Rep. Prog. Phys. 67, 1769 (2004).

[6] K. Sakimoto, Phys. Rev. A 65, 012706 (2001).

[7] S. Y. Ovchinnikov and J.H. Macek, Phys. Rev. A 71, 052717 (2005).

[8] N. Yamanaka and A. Ichimura, Phys. Rev. A 74, 012503 (2006).

[9] X. M. Tong, K. Hino, and N. Toshima, Phys. Rev. Lett. 97, 243202 (2006).

[10] X. M. Tong, T. Shirahama, K. Hino, and N. Toshima, Phys. Rev. A 75, 052711 (2007).

[11] J.S. Cohen, Phys. Rev. A 69, 022501 (2004).

[12] K. Sakimoto, Phys. Rev. A 74, 022709 (2006).

[13] J. S. Cohen, Phys. Rev. A 65, 052714 (2002).

[14] Y. Yamazaki (private communication).

[15] X. M. Tong and C. D. Lin, J. Phys. B 38, 2593 (2005).

[16] X. M. Tong and S. I. Chu, Phys. Rev. A 55, 3406 (1997).

[17] I. Shimamura, Phys. Rev. A 46, 3776 (1992). 